\title{
THE INFLUENCE OF MORPHOLOGY UPON TWO-TEMPERATURE STATEMENTS FOR CONVECTIVE TRANSPORT IN POROUS MEDIA
}

\author{
L. J. Gratton, V. S. Travkin, and I. Catton \\ Mechanical, Aerospace and Nuclear Engineering Department, University of California, Los \\ Angeles, CA 90024-1597
}

Transport models for forced, single phase fluid convection are reviewed for non-uniformly and randomly structured highly porous media. Special attention is given to the evaluation of a twotemperature energy model. For means of comparison, a one-temperature, effective thermal diffusivity model is developed, emphasizing local solid phase morphology using analytic techniques. Random characteristics of the porous medium are simulated by the use of regular and unspecified, pre-assigned solid phase morphologies. An overall coefficient of drag resistance is determined by implementing a multiple-regime superposition approach. Coefficient models are evaluated using the governing averaged transport equations set and solved numerically. Variability of the morphology descriptors is shown to potentially govern large fluctuations in transport parameter values and distributions. Results generally compare favorably among work by Koch and Brady; Fand and Thinakaran; Adnani, Raffray, Abdou, and Catton; and Watanabe.

Key Words: Highly porous media; Convective heat transfer; Two-temperature energy equation; Transport coefficients; Solid medium morphology

Description of the events occurring in a porous medium undergoing thermal and inertial phenomena can often be accomplished with acceptable error using averaged values of physical quantities. Kovalenko (1991) argues that heterogeneous real disperse media may be treated as continuous and the principal laws may be stated in terms of averaged quantities when the criterion

$$
\frac{\delta}{l} \ll 1
$$


is satisfied, where $*$ is the observed or assumed micro-scale inhomogeneity and 1 is the macro-scale. Koch and Brady (1985) note that the description of macro-transport, not transport by microscopic process, is the "primary interest." For such emphasis, assessment by averaged quantities is acceptable.

Treating a mechanical mixture as a homogeneous continuum entails the use of effective transport coefficients. Selection of effective transport coefficient models appropriate to the situation under study is most relevant under such treatment. Hence, "appropriate selection" is dictated by the matrix microstructure, which greatly influences both the transport coefficient magnitudes and spatial behavior of transport parameters. The current study analyzes both parameter magnitude and behavior involving a two-temperature energy statement, the values of the overall drag coefficient, $c_{d}$, the effective thermal conductivity for a one temperature, local thermal equilibrium energy statement, $k_{\text {eff }}$, and the heat transfer coefficient at interphase boundaries for the two-temperature energy statement, "\#.

Consideration is given to steady, two-dimensional momentum and heat transfer within a two-phase system. A flat channel is fully occupied by an unconsolidated highly porous medium composed of uniform packed spheres. Immediate interests lie in the prediction of phenomena pertaining to the case of large $\mathrm{k}_{\mathrm{s}} / \mathrm{k}_{\mathrm{f}}$ ratios, thus the specific case and physical parameter values considered best describe that of a steel spherical-bead matrix with incompressible air entering the interstices by forced, non-Darcy convection. Free convective heat/mass transfer processes are assumed negligible. Interphase boundaries are impermeable to the fluid. Wall channeling is determined to be inconsequential for the current bulk process evaluation using criteria established in experimental work by Fand and Thinakaran (1990). 


\section{ANALYSIS}

\section{Morphology Description}

Three packings with distinct characteristics are considered:

1.) Regular packing of solid spheres with assigned morphology characteristics (Fig. 1(a)),

2.) Simple cubic unit cell structure with solid spheres at the lattice nodes and exact expressions for the morphology functions (Fig. 1(b)),

3.) Bi-porous structure (depiction of a representative capillary in Fig. 1(c)).

Rigorous descriptions of the morphologies employed for the three structures are offered in Gratton et al. (1993).

\section{The Governing Equations}

The statement for momentum exchange is obtained by the simplification of a generalized twodimensional development for flow in a randomly homogeneous porous medium confined to a layer. Derivation of the generalized equation for developed flow in a monodisperse porous medium is performed by stochastic averaging and further detail is offered by Travkin and Catton (1992b and 1995). The consideration of a regular medium allows omission of the morpho-fluctuation terms, which account for solid-microstructure deviation from an idealized configuration. The hydrodynamic problem is further simplified by the assumption of no interphase penetration and the introduction of a closure process for the momentum equation involving the mean developed turbulent velocity averaged over the fluid portion of a representative elementary volume (Travkin and Catton, 1992a,b and 1995). The resulting governing statement for developed flow is given by,

$$
\begin{aligned}
\frac{\partial}{\partial z}\left[<m(z)>\left(v+\tilde{K}_{m}(z, \widetilde{\widetilde{u}}, \widetilde{b}, l)\right)\right. & \left.\frac{\partial \widetilde{\tilde{u}}(z)}{\partial z}\right]= \\
& \frac{c_{d}}{2} S_{w}(z) \stackrel{2}{\tilde{u}(z)}+\frac{1}{\rho_{f}} \frac{\partial(<m(z)>\widetilde{\bar{p}})}{\partial x},
\end{aligned}
$$


where the overall drag coefficient, $\mathrm{c}_{\mathrm{d}}$, is defined

$$
c_{d}=\frac{\left[c_{F L}(z, \tilde{\tilde{u}}) S_{w L}(z)+\tilde{c}_{d}(z, \widetilde{\tilde{u}}) S_{w T}(z)+c_{d p}(z, \widetilde{u}) S_{w p}(z)\right]}{S_{w}(z)} .
$$

The mean turbulent eddy viscosity, $Z_{\mathrm{m}}$, is determined from

$$
\tilde{K}_{m}(z)=C_{1}^{1 / 4} l(z) b^{1 / 2}(z)
$$

where $1(\mathrm{z})$ is the turbulent scale function, defined by the assigned porous medium structure, $\mathrm{C}_{1}$ is the turbulent exchange coefficient, and $b(z)$ is the mean turbulent fluctuation energy function, which satisfies the closure scheme

$$
\begin{gathered}
\tilde{K}_{m}(z)\left(\frac{\partial \tilde{u}}{\partial z}\right)^{2}+\frac{d}{d z}\left(\left(\frac{\tilde{K}_{m}}{\sigma_{b}}+v\right) \frac{d b(z)}{d z}\right)+\frac{f_{1}\left(c_{d}\right) \cdot S_{w}(z)}{<m>} \tilde{u}^{3} \\
-2 v\left(\frac{d}{d z}\left[b^{0.5}\right]\right)^{2}-\frac{g}{T_{a} \sigma_{T}}\left[\tilde{K}_{m} \frac{\partial \widetilde{T}}{\partial z}\right]=C_{1} \frac{b^{2}}{\tilde{K}_{m}} .
\end{gathered}
$$

The turbulent scale function, $1(\mathrm{z}$ ), is calculated as a function of morphology descriptors (see Travkin and Catton, $1992 \mathrm{a}$ and b).

The heat transfer problem is governed by the generalized two-dimensional, two-temperature statement for energy transport in a porous layer, providing one governing equation in the fluid phase and a second in the immobile, inpenetrable phase (Travkin and Catton, 1992b and 1995). The closure process given in work by Travkin and Catton (1992a,b and 1995) allows reduction of the integral terms to differential terms with dynamic coefficients. Restricting attention to regular media further simplifies the problem in a manner analogous to that seen for the hydrodynamic statement. The two-dimensional governing equation in the fluid phase subsequently assumes the form, 


$$
\begin{aligned}
\rho_{f} c_{p f}<m>\widetilde{\widetilde{u}}(z) \frac{\partial \widetilde{T}(x, z)}{\partial x}=\frac{\partial}{\partial z} & {\left[<m>\tilde{K}_{T}(z) \frac{\partial \widetilde{T}(x, z)}{\partial z}\right] } \\
& +\widetilde{\alpha}_{T}(z) S_{w}(z)\left[T_{s}(x, z)-\widetilde{T}(x, z)\right],
\end{aligned}
$$

where $(\mathrm{x}, \mathrm{z}) 0) \mathrm{S}_{\mathrm{f}}$, and becomes the following in the solid phase

$$
\begin{aligned}
\frac{\partial}{\partial z}\left[(1-<m(z)>) K_{S T}(z) \frac{\partial \widehat{T}_{s}(x, z)}{\partial z}\right]= \\
\tilde{\alpha}_{r}(z) S(z)\lceil\widehat{T}(x, z)-\widetilde{T}(x, z)\rceil .
\end{aligned}
$$

when (x,z) 0$) S_{s}$. The thermal eddy conductivity, H $_{T}$, for Eq. (6), is determined

$$
\widetilde{K}_{T}=\rho_{f} c_{p, f} \widetilde{K}_{m}+k_{f}
$$

valid for $\operatorname{Pr}_{\mathrm{T}} \bullet 1$. For treatment under a local-thermal equilibrium assumption, a simplistic energy model is governed by a one-temperature equation for a continuum with assumed homogeneity and effective thermo-physical properties,

$$
\begin{aligned}
& \rho_{\text {eff }} c_{p, e f f}<m(z)>\widetilde{u}(z) \frac{\partial<T(x, z)>}{\partial x}= \\
& \underline{\partial}\left[\begin{array}{l}
\left\langle m(z)>k_{. 凶} \underline{\partial<\bar{T}(x, z)>}\right\rceil .
\end{array}\right.
\end{aligned}
$$

which is effective over the entire domain $(x, z) 0) \mathrm{S}$. The effective properties, $\mathrm{D}_{\mathrm{eff}}$ and $\mathrm{c}_{\mathrm{p} \text {, eff }}$ are defined 


$$
\begin{aligned}
\rho_{e f f} & =\frac{\rho_{f}}{h} \int_{0}^{h} m(z) d z+\rho_{s}\left\lfloor 1-\frac{1}{h} \int_{0}^{h} m(z) d z\right\rfloor, \\
c_{p, e f f} & \left.=\frac{c_{p, f}}{h} \int_{n}^{h} m(z) d z+c_{p, s} \mid 1-\frac{1}{h} \int_{n}^{h} m(z) d z\right] .
\end{aligned}
$$

Equations (2), (5), (6), and (7) are subjected to the following boundary conditions

$$
\begin{aligned}
& z=0: \widetilde{u}=0, \frac{\partial b}{\partial z}=0, \tilde{K}_{m}=0, Q_{0}=-\tilde{K}_{T} \frac{\partial \widetilde{T}}{\partial z}, Q_{0}=-K_{s} \frac{\partial \widehat{T}_{s}}{\partial z}, \\
& z=h: \frac{\partial \widetilde{u}}{\partial z}=0, \frac{\partial b}{\partial z}=0, \frac{\partial \widetilde{T}}{\partial z}=0, \frac{\partial \widehat{T}_{s}}{\partial z}=0,
\end{aligned}
$$

corresponding to constant heat flux, hydrodynamic no-slip, and no variation in the magnitude of the turbulent fluctuation energy at the wall. Symmetry conditions for all profiles are imposed at the channel mid-plane. Use of the one-temperature model (Eq. (9)) merely requires the use of boundary conditions similar to those used for the fluid-phase energy equation in the two-temperature statement

$$
\begin{aligned}
& z=0: Q_{0}=-k_{e f f} \frac{\partial<\bar{T}>}{\partial z}, \\
& z=h: \frac{\partial<\bar{T}>}{\partial z}=0
\end{aligned}
$$

For means of comparison with the one-temperature equation results, an equivalent local temperature is defined for the two-temperature system by volume fraction weighting

$$
T_{w t d}=<m(z)>\widetilde{T}(z)+[1-<m(z)>] \widehat{T}_{s}(z)
$$

\section{The Overall Drag Coefficient}


Various models and correlations are offered in determination of the overall drag coefficient, $\mathrm{c}_{\mathrm{d}}$, used in the hydrodynamic equation (Eq. (2)). Travkin and Catton (1992a and b) discuss the

$$
\frac{\Delta p}{L}=f\left(\frac{S_{W}}{<m>}\right)\left(\frac{\rho_{f} \vec{u}^{2}}{2}\right)
$$

applicability in the bulk laminar regime of a commonly used experimental relationship

for the pressure drop in packed beds, yielding a theoretical drag coefficient approximation for constant or large-scale nonuniform porosity

$$
c_{d} \simeq f,
$$

where $\mathrm{f}$ is the hydrodynamic resistance coefficient for packed beds taken from experimental correlations. The sensitivity of Eq. (15) to the variation of, at minimum, the morphology functions $\mathrm{m}$ and $\mathrm{S}_{\mathrm{w}}$ is noted. Consideration is now given to calculation of $\mathrm{c}_{\mathrm{d}}$ in the combined and turbulent regimes by similar treatment.

Watanabe (1989) suggests a formula for the overall drag resistance of a packed bed consisting of spheres

$$
c_{d} \approx f=\left[\frac{25}{12}(1-<m(z)>)\right] c_{d, s p h}
$$

where $c_{d, \text { sph }}$ is the coefficient of drag resistance for a single isolated bead of diameter $d_{p}$. To implement Eq. (16), a particle Reynolds number $\left(\operatorname{Re}_{R}\right)$ dependent family of correlations is assembled from a review by Boothroyd (1971) to approximate the mean drag resistance $\left(\mathrm{c}_{\mathrm{d}, \mathrm{sph}}\right)$ over a single, isolated sphere in various flow regimes. The various correlations for $c_{d, s p h}$ and the respective $\operatorname{Re}_{R}$ ranges of usage are provided in Table 1. 


\begin{tabular}{|c|c|c|}
\hline Flow Regime & \multicolumn{2}{|c|}{ Expression for the Particle Drag Coefficient } \\
\hline $\operatorname{Re}_{\mathrm{R}} \# 0.2$ & $c_{d, s p h}=\frac{24}{R e_{\psi}}$ & (17) \\
\hline $0.2<\operatorname{Re}_{\mathrm{R}} \# 3.0$ & $c_{d, s p h}=\frac{24}{R e_{\psi}}\left\lfloor 1+\frac{3}{16} R e_{\psi}\right\rfloor$ & (18) \\
\hline $3.0<\operatorname{Re}_{\mathrm{R}}<400$ & $c_{d, s p h}=\frac{24}{R e_{\psi}}\left[1+0.15 R e_{\psi}^{0.687}\right]$ & (19) \\
\hline $400 \# \mathrm{Re}_{\mathrm{R}} \# 500$ & $=\frac{24}{R e}\left[1+0.197 R e_{\psi}^{0.63}+0.002\right.$ & (20) \\
\hline $500<\operatorname{Re}_{\mathrm{R}}<2\left(\bigcup^{5}\right.$ & $c_{d, s p h}=0.44$ & (21) \\
\hline $\operatorname{Re}_{\mathrm{R}} \$ 2 @ 0^{5}$ & $c_{d, s p h}=0.1$ & $(22)$ \\
\hline
\end{tabular}

Table 1. Expressions and ranges of validity used to determine the drag coefficient for a single, isolated sphere.

Equations (17)-(22) provide an effective means of calculating the flow resistance for packed beds when combined with Watanabe's correction (Eq. (16)).

Additional empirical expressions may be used to close the hydrodynamic equation (Eq. 2). Table 2 provides a brief summary of the remaining globular morphology drag coefficient models used in the analysis. For spherical obstacles, the Reynolds number based upon characteristic pore size is defined

$$
R e_{c h}=\frac{2}{3} \frac{<m>}{(1-<m>)} \frac{\widetilde{u} d_{p}}{v}
$$

Equation (23) is used to define the parameter $\mathrm{Re}_{\mathrm{ch}}$ used in Eqs. (24), (25) and (26). Use of Eq. (23) 
in lieu of the Reynolds number originally defined by Klenov and Matros (1990) results in the modified form given by Eq. (25). The Klenov and Matros (1990) formula is experimentally determined for $0.3 \#<\mathrm{m}>\# 0.4$ and $\mathrm{O}(2) \# \mathrm{Re}_{\mathrm{ch}} \# 1700$ for spherical particles.

\begin{tabular}{|c|c|}
\hline Relation & Expression for the Overall Drag Coefficient \\
\hline \hline Ergun (1952) & $\simeq f=\frac{A}{R e_{c h}}+B, A=33.33, B=c$ \\
\hline Klenov and Matros (1990) & $\simeq f=\frac{A}{R e_{c h}}+B, A=36.3, B=$ \\
\hline Fand and Thinakaran (1990) & $c_{d} \simeq f=\frac{2}{9} \frac{A}{R e_{c h}}+\frac{B}{3}$
\end{tabular}

Table 2. Additional expressions used to determine the overall drag coefficient for sphere packed beds.

Correlations are presented by Fand and Thinakaran (1990) for randomly packed cylinders

$$
10^{-5}<R e_{\psi} \lesssim O(3)
$$

enclosing solid matrices composed of uniform spheres, valid over the range of Reynolds numbers

whereby the modified friction factor, $\mathrm{f}^{\prime}$, is given by

$$
f^{\prime}=\left(\frac{-d P}{\rho_{f} \widetilde{\widetilde{u}}}\right) \frac{<m>^{3}}{(1-<m>)}=A \frac{(1-<m>)}{R e_{\psi}}+B
$$

Manipulation of Eq. (28), using Eqs. (14), and (23) provides the alternate form of Fand and 
Thinakaran's (1990) empirical correlation for $\mathrm{f}$ in terms of $\mathrm{Re}_{\mathrm{ch}}$ which is presented in Eq. (26). The coefficients A and B in Eq. (26) are flow regime dependent (e.g. Darcy, Forchheimer, turbulent), whereupon the reader is directed to the reference for the determined numerical values.

Progression to capillary channel models extends the number of available formulae for $\mathrm{c}_{\mathrm{d}}$. For a tube morphology consisting of straight circular channels of equivalent diameter, Travkin and Catton (1992a and $\mathrm{b})$ show that

$$
c_{d}=\tilde{c}_{d} \simeq \frac{\lambda}{4}
$$

If it is assumed that the characteristics of the path through the voids in the interstices of neighboring spheres have equivalent expression in a system of straight parallel smooth tubes, Nikuradse's (1950) formula may be used

$$
c_{d}=\tilde{c}_{d} \simeq \frac{\lambda}{4}=\frac{1}{4}\left(0.0032+0.221 R e_{c h}^{-0.237}\right) .
$$

Should the added effects of wall roughness in the tubes better simulate those corresponding to the morphology under consideration, then Nikuradse's (1950) formula for rough tubes provides

$$
c_{d}=\tilde{c}_{d} \simeq \frac{\lambda}{4}=\frac{\left(\frac{1}{\left[0.87 \ln \left(d_{c h} / 2 h_{r}\right)\right.}+1.74\right)^{2}}{4} .
$$

For Eq. (31), $d_{\text {ch }}$ is defined

$$
d_{c h} \equiv \frac{4<m>}{S_{w}}
$$

rendering the general definition of $\mathrm{Re}_{\mathrm{ch}}$ for Eq. (30), in lieu of the more specific form used hitherto (Eq. (23)) 


$$
R e_{c h}=\frac{\widetilde{u} d_{c h}}{v}
$$

Expression of $\mathrm{c}_{\mathrm{d}}$ by means of Eq. (3) presupposes knowledge of micro-scale phenomena. Existence of experimental work quantifying boundary-layer characteristics at interphase regions within porous media is unbeknownst to the authors. Furthermore, little work has been done in quantifying the relative contribution of form drag to the overall resistance in porous media. Fand et al. (1987) note the absence of hitherto published work upon the subject of flow resistance in porous media which distinguish between laminar and turbulent Forchheimer regimes, citing their original work as "particularly interesting" upon that basis. Their experimental findings for a cylindrical tunnel with random packings of spheres include the observation of a laminar Forchheimer regime, 5 \# $\operatorname{Re}_{R} \#$ 80, a transition Forchheimer regime, $80<\mathrm{Re}_{\mathrm{R}} \# 120$, and a turbulent Forchheimer regime, $\mathrm{Re}_{\mathrm{R}}>$ 120. The foregoing allow approximate treatment of Eq. (9) for the combined and turbulent regimes if the portions of the fluid-solid interface experiencing laminar, transition and turbulent boundary layer or recirculatory flow can be identified. Assuming that the near-wall flow can be characterized in such a manner, Eq. (3) acquires the form

$$
c_{d}=\left(\tilde{c}_{d}+c_{d p} \frac{S_{w p}(z)}{S_{w}(z)}\right)
$$

The relative contributions of form and friction drag in flows through monodisperse, unconsolidated porous media are discussed in Gratton et al. (1993).

The drag coefficient for the bi-porous medium assumption is obtained by the linear superposition of the Nikuradse (1950) rough tube formula (with an assigned roughness height $h_{r}=d_{c h} / 10$ ) and a pressure drag component relationship (Gratton et al., 1993). Calculation of the pressure drag term is accomplished using a correlation by Tsuji et al. (1985) for a bi-porous medium consisting of spherical inclusions in cylindrical conduits 


$$
c_{d p}=\frac{\alpha(1-<m>)^{\beta}}{d_{c h} S_{w p}}
$$

where " $=2.58$ and $\$=0.75$. Details are offered by Gratton et al. (1993)

\section{The Effective Thermal Conductivity}

A substantial amount of literature is available containing methods to formulate the effective thermal conductivity in porous media for use in conjunction with energy equations assuming local thermal equilibrium. Less attention, however, is given to methods of determining the lateral component of the thermal conductivity, $\mathrm{k}_{\mathrm{eff}}$, in systems with large $\mathrm{k}_{\mathrm{s}} / \mathrm{k}_{\mathrm{f}}$ ratios and high void fractions. Adnani et al. (1989) suggest

$$
k_{e f f}=k_{0}+\left(\frac{0.43}{(1-<m>)}\right) d_{p} \rho_{f} C_{p, f}<m>\widetilde{\widetilde{u}}
$$

where $\mathrm{k}_{0}$ is the effective stagnant thermal conductivity for the packed bed. Experimental data from Adnani et al. (1989) shows that for metal particle/gas systems, with $\mathrm{k}_{\mathrm{s}} / \mathrm{k}_{\mathrm{f}} \cdot 1410$, the stagnant term is found

$$
k_{0}=15 k_{f}
$$

Koch and Brady (1985) find the overall conduction in a fixed bed to be the linear combination of contributions from the molecular conduction, the solid/fluid differences in conductivity, and the effects arising from convection. The authors develop, by means of asymptotic analysis, particle Peclet number dependent expressions for the transverse component of the effective thermal conductivity in media with low solid volume fraction 


$$
\begin{gathered}
k_{e f f}=k_{f}\left(1+3\left(\frac{\frac{k_{s}}{k_{f}}-1}{\frac{k_{s}}{k_{f}}+2}\right)(1-<m>)+\frac{\sqrt{2}}{60} \frac{P e_{r}^{2}}{(1-<m>)^{1 / 2}}\right), \\
P e_{r} \ll(1-<m>)^{1 / 2} \ll 1, P e_{k} \ll 1,
\end{gathered}
$$

where $\mathrm{Pe}_{\mathrm{r}}$ is the Peclet number founded upon particle radii,

$$
P e_{r}=\frac{\widetilde{\widetilde{u}} d_{p}}{a_{f} 2}
$$

and $\mathrm{Pe}_{\mathrm{k}}$ is the Peclet number with respect to the Brinkman screening length,

$$
P e_{k}=\frac{\widetilde{\widetilde{u}} d_{p}}{a_{f}}\left(\frac{1}{18(1-<m>)}\right)^{1 / 2}
$$

A second $\mathrm{Pe}_{\mathrm{r}}$ regime requires calculation of the effective thermal conductivity by

$$
\begin{gathered}
k_{e f f}=k_{f}\left[1+3 \frac{\left(\frac{k_{s}}{k_{f}}-1\right)}{\left(\frac{k_{s}}{k_{f}}+2\right)}(1-<m>)+\frac{2}{9(1-<m>)} \times\right. \\
\left\{\frac{1}{4}+\frac{3}{4}\left|P e_{k}\right|^{-1}-\frac{3}{4}\left|P e_{k}\right|^{-2}+\frac{3}{2}\left(\left|P e_{k}\right|^{-3}-\frac{1}{2}\left|P e_{k}\right|^{-1} \ln \left[\left|P e_{k}\right|+1\right]\right)\right\} \\
(1-<m>)^{3 / 4} \ll P e_{r} \ll 1
\end{gathered}
$$


Within the interval

$$
(1-<m>) \ll P e_{r} \ll 1, P e_{k} \gg 1,
$$

$\mathrm{k}_{\text {eff }}$ is described

$$
k_{e f f}=k_{f}\left[1+\frac{3 \sqrt{2}}{8}(1-<m>)^{1 / 2}\right] .
$$

The last of the Koch and Brady formulae is expressed

$$
\begin{gathered}
k_{e f f}=k_{f}\left[1+\frac{63 \sqrt{2}}{320}(1-<m>)^{1 / 2}\left|P e_{r}\right|\right], \\
P e_{r} \gg 1 .
\end{gathered}
$$

Alternately, $\mathrm{k}_{\text {eff }}$ may be evaluated as a linear combination of contributions from molecular diffusion in both the solid and fluid phases, and turbulent dispersion in only the fluid phase. If transport properties are viewed as the volume-fraction weighted influences of the independent contributions, an appropriate formulation is

$$
k_{e f f}=\left(\rho_{f} c_{p, f} \widetilde{K}_{m}+k_{f}\right) \frac{1}{h} \int_{0}^{h} m(z) d z+k_{s}\left[1-\frac{1}{h} \int_{0}^{h} m(z) d z\right] \text {. }
$$

\section{The Local Heat Transfer Coefficient}

Calculation of the void averaged heat transfer coefficient, "\#, necessary for an energy statement of the form given in Eqs. (6) and (7), is performed using assorted formulae. Expressions used to obtain the heat transfer coefficient in this study are presented in Table 3. 


\begin{tabular}{|c|c|c|}
\hline Relation & Expression for the Heat Transfer & \\
\hline Aarov et al. (1979) & $\left.\tilde{t}_{T}\right|_{--\cap H}=\alpha_{w}=\frac{k_{f}}{d_{\text {sh }}}\left[0.155 \operatorname{Re}_{c h}^{0.75} \operatorname{Pr}^{1 / 3}\right.$ & $(45)$ \\
\hline Kokorev et al. (1987) & $\tilde{\alpha}_{T}=\frac{k_{f}}{d_{p}}\left(0.14\left(c_{d} R e_{c h}^{3}\right)^{1 / 4} \operatorname{Pr}^{1 / 3}\right)$ & $(46)$ \\
\hline Kokorev et al. (1987) & $T_{T}=\frac{k_{f}}{d_{p}}\left(4+\left(133 \operatorname{Re}_{c h}^{2}+2.33 \operatorname{Re}_{c h}^{3}\right)^{1 / 4} \operatorname{Pr}^{1 /}\right.$ & $(47)$ \\
\hline $\begin{array}{c}\text { Heat Exchanger } \\
\text { Design Handbook } \\
\text { (1983) }\end{array}$ & $\begin{array}{c}\tilde{\alpha}_{T}=\frac{[1+1.5(1-<m(z)>)}{d_{p}} \\
\left.1.664 \operatorname{Re}_{\psi}^{1 / 2} \operatorname{Pr}^{1 / 3}\right)^{2}+\left(\frac{0.037 \operatorname{Re}_{\psi}^{0.8} \operatorname{Pr}}{\sqrt{1+2.443 \operatorname{Re}_{\psi}^{-0.1}\left(\operatorname{Pr}^{\prime}\right.}}\right.\end{array}$ & (48) \\
\hline
\end{tabular}

Table 3. Expressions used to determine the heat transfer coefficient for sphere packed beds.

Aarov et al. (1979) developed an equation for use in the near-wall region in granular beds. Thermal convection from the wall to the fluid in the vicinity of the wall is described by Eq. (45) in the range

$$
150 \leq R e_{c h} \leq 4000 .
$$

Kokorev et al. (1987) present a correlation between drag coefficient and heat transfer coefficient, given by Eq. (45) for turbulent flow in a porous medium consisting of spherical beads, granular constituents or fibrous material. Additionally, Kokorev et al. (1987) introduce a specific form of Eq. (46), suggested for 10 \# $\mathrm{Re}_{\mathrm{ch}} \# 10^{6}$ and given by Eq. (47). The last of the formulae considered, 
Eq. (48), is a correlation highly dependent upon local morphological and flow quantities (Heat Exchanger Design Handbook, 1983).

\section{Implementation and Numerical Solution}

The governing equations are non-dimensionalized as presented in Travkin and Catton (1992b). Solutions to the equation set are obtained utilizing a finite difference scheme for non-linear grid spacing and discontinuous coefficients as described in Travkin (1981, 1984, 1985 and 1987). Solutions of the equation set are obtained on an IBM 3090.

Numerical solution of the set of equations is performed using the presented models for the overall drag coefficient, the effective thermal conductivity for Eq. (9) and the heat transfer coefficient for Eqs. (6) and (7). Solutions are obtained for both the unspecified, constant porosity and the simplecubic local morphology descriptions. The effects of a bi-porous solid medium upon the transport processes are approximated in the numerical study through their impact on the hydrodynamic field by the usage of Eq. (35) with both the constant and periodic morphology characterizations. The channel boundary drag coefficient values are determined by a method independent of the resistance model chosen to avoid the potential for singularities due to zero wall velocity. Because current interests are focused upon an air/steel system for the fluid/solid combination, the conductivity ratio, $\mathrm{k}_{\mathrm{s}} / \mathrm{k}_{\mathrm{f}}=1490$ is appropriately chosen.

\section{RESULTS AND DISCUSSION}

\section{The Overall Drag Coefficient and the Velocity Field}

Distinct differences among the capillary and globular models are observed for both the unspecified and simple cubic morphologies at $\mathrm{d}_{\mathrm{p}} / \mathrm{h}=0.05$ and $\mathrm{x} / \mathrm{h}=3.0$. Figure 2(a) illustrates local drag coefficient profiles over one-quarter of the layer corresponding to a uniform void fraction $(<\mathrm{m}>=$ 0.8 ) for the eight models. The Nikuradse formulae predict approximately two orders of magnitude below the globular models, which is consistent with the greater flow resistance offered by solid obstacles as compared to straight channels. The equations suggested by Boothroyd (1971) (Eqs. (17)-(22)), when not corrected for packed bed applications with Watanabe's expression (Eq. (16)), 
slightly overpredict the flow resistance in comparison to the remaining globular models.

The more detailed local description of the layer's local morphological characteristics in the case of the cubic geometry illustrates the effects of local structure variation upon the models. The cubic yields $\mathrm{m}_{0}=0.48$. Again, results are monitored at $\mathrm{d}_{\mathrm{p}} / \mathrm{h}=0.05$ and $\mathrm{x} / \mathrm{h}=3.0$. The capillary resistance models differentiate themselves from the globular models by comparative underpredictions of anywhere from 2 to 4 orders of magnitude. Though the uncorrected Boothroyd suggestions are in better agreement with the remaining globular model averages than in the instance of an unspecified local structure $(<\mathrm{m}>=0.8)$, they are incapable of capturing the local value oscillations owing to the absence of any explicit morphology dependence. Watanabe's correction, however, brings the singlesphere models into strict agreement with the remaining globular expressions for the cubic lattice morphology. Nikuradse's rough tube formula, aside from being physically imprecise for the specific application, poses numerical problems related to small values of local void fraction and the inverse natural logarithmic dependence of the formula. The problems with the Nikuradse formulae give even greater credence to the necessity to properly account for microstructural effects by the choice of a representative model of the medium and appropriate coefficient models.

Figure 3(a) displays velocity profiles corresponding to the respective drag coefficient models for the case of constant void fraction $(<\mathrm{m}>=0.8)$ and $\mathrm{d}_{\mathrm{p}} / \mathrm{h}=0.05$. Again, the monitoring line is located at a dimensionless longitudinal distance of 3.0 for all eight resistance models. The capillary (Nikuradse) models tend to overpredicit the bulk velocity and the single sphere models predict conservatively when compared to the globular models. Though approximate in its present form, the multiple contribution approach (superposition of Eqs. (31) and (35)) serves to bring the capillary models into agreement with the experimentally determined globular expressions for packed beds by modifying the straight tube assumption to that of a bi-porous medium comprised of capillary tubes with included spherical obstructions (Fig. 1(c)). The agreement among the globular and bi-porous drag coefficient expressions persists when viewed as a function of the Reynolds number or the dimensionless pressure gradient. Figure $3(\mathrm{~b})$ shows the variation of the mean drag coefficient, $\mathrm{c}_{\mathrm{d}, 0}$, with respect to $\mathrm{Re}_{\mathrm{Q}}$. The most notable feature is the constancy of the multiple contribution formulation, caused by the choice of Nikuradse's rough tube formula (which is merely a function of geometric parameters) for $\#_{t}$. Order of magnitude accuracy is maintained through the combined and well into the turbulent regions among the Klenov and Matros, Fand and Thinakaran, Watanabe 
and bi-porous results.

Figure 4(a) illustrates the non-dimensional velocity profiles in the lower quarter-channel for the case of the cubic geometry $\left(\mathrm{m}_{0}=0.48\right)$ and $\mathrm{d}_{\mathrm{p}} / \mathrm{h}=0.05$ at a dimensionless longitudinal distance of 3.0. With the exception of the Boothroyd and Nikuradse rough tube formulae, variability of the resistance values is manifested as oscillatory velocity fields. As with the constant porosity case, the capillary and globular models clearly distinguish themselves, as do the essentially uniform profiles associated with the Ergun, Nikuradse rough tube and uncorrected Boothroyd formulae. Figure 4(b) shows that the multiple contribution approach predicts mean drag coefficient values of the same order of magnitude and displays similar flow regime dependence as three of the globular models over a large range of $\mathrm{Re}_{\mathrm{R}}$ for the cubic lattice.

\section{The Effective Thermal Conductivity and the Temperature Field}

Figure 5 displays dimensionless effective thermal conductivity results for $<\mathrm{m}>=0.8$, again assigning $\mathrm{d}_{\mathrm{p}} / \mathrm{h}=0.05$. Values are monitored at a dimensionless longitudinal coordinate of 3.0. The Adnani et al. model (Eq. (36)) and Eq. (44) give order of magnitude agreement, while the pair distinguish themselves from the Koch and Brady model (Eqs. (38)-(43)) by an order of magnitude. The Koch and Brady model shows a near-wall increase in the predicted coefficient values. The increase arises from the regime where $\mathrm{k}_{\text {eff }}$ goes as $1 / \mathrm{Pe}_{\mathrm{k}}$ (Eq. (41)). Conversely, the Adnani et al. model's near-wall descent of the calculated values is attributable to its linear fluid velocity dependence.

Figure 6 illustrates results for the case of cubic geometry, with the same boundary conditions and geometric parameters as given above, except $\mathrm{m}_{0}=0.48$. The Koch and Brady and Adnani et al. formulae respond differently to porosity variation. The Koch and Brady formulae are dependent upon solid fraction $(1-<\mathrm{m}>$ ) and return the highest values where $<\mathrm{m}>$ is of lowest value, despite small $\mathrm{Pe}_{\mathrm{r}}$ in regions of low $<\mathrm{m}>$. The Adnani et al. equation includes a convective influence term which is dependent upon the Darcy velocity, returning the highest values in regions of maximum void. Though Eq. (44) makes use of layer averaged porosities, minor oscillations are introduced by means of the turbulent eddy diffusion term, $\mathrm{D}_{\mathrm{f}} \mathrm{c}_{\mathrm{p}, \mathrm{f}}$, which depends upon the turbulent scale and the mean turbulent fluid velocity, both very sensitive to the microstructure. However, the 
oscillations are dwarfed in comparison to the magnitude of the solid contribution, especially for the moderate $\mathrm{m}_{0}$ used.

Figure 7 provides the corresponding dimensionless temperature profiles for $<\mathrm{m}>=0.8, \mathrm{~d}_{\mathrm{p}} / \mathrm{h}=0.05$, a dimensionless longitudinal station of 3.0, and $\mathrm{Q}_{0}=20\left[\mathrm{~W} / \mathrm{m}^{2}\right]$. The curves associated with the Koch and Brady, Adnani et al., and Eq. (44) models are identical, as is the weighted temperature (Eq. (13)) profile from the two-temperature model results associated to the use of Eq. (46) for the turbulent heat transfer coefficient. Agreement among the one-temperature model profiles (Eq. (9)) is largely attributable to the manner in which $D_{\text {eff }}$ and $c_{p, \text { eff }}$ are employed and the dominating influence of the solid contribution to energy transport. Figure 8 gives the non-dimensional mean effective thermal conductivity as a function of Peclet number. The Koch and Brady and Adnani et al. formulae keep roughly one order of magnitude difference over a large range of Pe. Equation (44) is effectively constant due to the dominant influence of the solid-side (steel bead) heat transfer as contrasted with the turbulent dispersion contribution.

Despite the disagreement among the average values returned by the three effective conductivity models, the temperature profiles for the simple cubic morphology case are identical in the bulk, varying only in the near-wall region (Fig.9(a)) under the conditions $\mathrm{m}_{0}=0.48, \mathrm{~d}_{\mathrm{p}} / \mathrm{h}=0.05, \mathrm{x} / \mathrm{h}=3.0$, and $\mathrm{Q}_{0}=20\left[\mathrm{~W} / \mathrm{m}^{2}\right]$. All of the one-temperature treatments underpredict with respect to the twotemperature model. Unlike the case of the unspecified morphology (Fig. 7), the boundary temperature gradients vary drastically among the one-equation models when used with a local morphology description. The oscillatory nature of the effective conductivity models is not appreciably imparted upon the temperature fields, a severe limitation imposed by the use of the onetemperature equation. Figure 9(b) displays the weighted temperature (Eq. (13)) resulting from the two-temperature model for the same physical situation. The oscillatory nature of the temperature field is captured, though less pronounced than if either the solid or fluid phase temperatures predicted by the two-temperature model are viewed independently. Additionally, there exists a predicted temperature discrepency of approximately 10 dimensionless degrees throughout the layer between the one- and two-temperature analyses. Explanation of the magnitude disagreement between the one- and two- temperature models, especially for large $\mathrm{k}_{\mathrm{s}} / \mathrm{k}_{\mathrm{f}}$ ratios, is offered in Travkin and Catton (1995). Behavior of the dimensionless layer-averaged effective conductivity values, when used with the simple cubic morphology, over a range of Pe is given in Fig. 9(c). The Koch 
and Brady model predictions remain roughly unchanged as compared to the unspecified morphology case, while the magnitudes of the averaged values from the Adnani et al. model and Eq. (44) show a one-to-two order of magnitude increase over the unspecified morphology consideration over a range of $\mathrm{Pe}$.

\section{The Local Heat Transfer Coefficient}

Figure 10(a) displays layer averaged turbulent Nusselt number values as determined by the Kokorev et al. formulae (Eqs. (46)-(47)) and Eq. (48) for the periodic morphology consideration with the physical situation identical to that modelled in the effective conductivity trials. Wall values calculated by the Aarov et al. model (Eq.(45)), are not displayed. The value $\mathrm{c}_{\mathrm{d}}$ used in Eq. (46) is determined using the Klenov and Matros expression (Eq. 25). The general form of the Kokorev et al. formula shows a minor indentation at local maxima, as does Eq. (48). The inflections are induced by local morphology variations through the parameter $d_{c h}$ in the case of Eq. (46) and a vanishing fluid velocity magnitude in both instances within regions of $<\mathrm{m}>\bullet 0$. Equation (47) shows little sensitivity due to the use of averaged quantities. The fluid temperature profiles associated with the heat transfer coefficient models (Fig. 10(b)) are similar in the bulk, with the greatest differences among the models appearing in channel boundary regions. The variation of the temperature distributions results in dissimilar gradients at the channel boundary. All three models do contribute to the capture of oscillations in the fluid temperature field. Figure 11(a) illustrates the behavior of the mean turbulent Nusselt number over varying Peclet number for the constant porosity regular morphology. The figure provides insights concerning the relative contributions of turbulent convection and turbulent eddy conduction. At high porosity, the latter takes an increasingly influential role, accounting for the reduction in $\mathrm{Nu}_{\mathrm{T}}$ observed for particle Pe above 100. At moderate $\mathrm{m}_{0}$, no such decline is observed over most of the same range of Pe (Fig. 11(b)). Additionally, the sensitivity of the Kokorev et al. formula to the choice of drag coefficient model is made obvious. The relative difference in the magnitudes of $\mathrm{Nu}_{\mathrm{T}}$ between Eqs. (46) and (47) for both morphology considerations is maintained, if not increased, over the Pe ranges considered. The magnitude difference is maintained explicitly through the parameter chosen for $\mathrm{c}_{\mathrm{d}}$, and implicitly through the fluid velocity magnitude. Again, failure to properly account for matrix microstructure can severely 
effect the reliability of the results because of the implicit morphological sensitivities of the expressions.

\section{CONCLUSIONS}

Turbulent transport equations are solved numerically in two dimensions for single-phase convective momentum and heat transfer in a flat channel. Both a one-temperature and a twotemperature energy statement are employed to determine the heat transfer. The transport equations are found to be sensitive to both the type of morphology description and the precision of the transport coefficient models.

The coefficient models for either the drag coefficient, effective thermal conductivity or heat transfer coefficient predict with varying magnitude and local behavior among themselves for identical physical situations. Regarding the drag coefficient, the capillary and globular models are shown to provide estimates which may vary by more than an order of magnitude, resulting in similar disparities in the predicted velocities. A multiple contribution formulation for the overall drag coefficient is capable of modifying the capillary structure model to that of a bi-porous medium such that better agreement with the globular predictions is obtained. Differences among the effective thermal conductivity models used in the one-temperature statement and the heat transfer coefficient models used in the two temperature statement severely impact the calculated temperature gradients at the channel boundaries in most cases.

Differences are also observed between the one- and two-temperature energy equations. In the bulk, the local thermal equilibrium statement proves somewhat insensitive to the morphology description and the effective conductivity model as a result of the large $\mathrm{k}_{\mathrm{s}} / \mathrm{k}_{\mathrm{f}}$ ratio employed. The onetemperature model shows excellent agreement with a weighted temperature from a two-temperature energy statement at high void fraction, mediocre agreement at moderate void fraction.

The effects of providing a locally detailed morphology description in the case of the simple cubic geometry incorporate both similarities and differences as compared to the commonly employed uniform, unspecified morphology description. The general Re (or Pe) dependent behavior of the layer averaged drag coefficient and effective thermal conductivity exhibits little variation. However, local behavior among the drag coefficient, effective thermal conductivity or heat transfer coefficient 
models is diverse between the simple cubic and unspecified morphology characterizations. Additionally, differences between the two morphologies are observed in the general behavior of the layer averaged Nusselt number over a range of Pe, though the large porosity value chosen for the unspecified morphology is thought to contribute to this dissimilarity.

The necessity for adequate description of morphological and phenomenological effects by the careful choice of governing equations and transport coefficient models is evident for modeling transport processes in porous media. However, the descriptive capabilities of a particular coefficient model are often limited or the coefficient model assumes an over-simplified morphological structure. The multiple contribution approach is capable of partially resolving such problems. Additionally, at low and moderate void fractions, a two-temperature energy statement not only predicts temperatures of dissimilar magnitude, but provides superior local resolution of the scalar field as compared to a one-temperature statement.

\section{ACKNOWLEDGMENTS}

This work was sponsored by the United States Department of Energy Grant No. DE -FG03 89ER14033 A002.

\section{NOMENCLATURE}

a thermal diffusivity $\left[\mathrm{m}^{2} / \mathrm{s}\right]$

$\mathrm{b}=(1 / 2) \overline{u_{i}^{\prime} u_{i}^{\prime}}$, mean turbulent fluctuation energy $\left[\mathrm{m}^{2} / \mathrm{s}^{2}\right]$

$\mathrm{C}_{1} \quad$ constant in Kolmogorov turbulent exchange coefficient correlation [-]

\# mean skin friction coefficient over the turbulent area of $\mathrm{B}_{\mathrm{w}}$ [-]

$\mathrm{c}_{\mathrm{d}} \quad$ mean drag resistance coefficient in the REV [-]

$\mathrm{c}_{\mathrm{d}, \mathrm{sph}} \quad$ drag resistance coefficient upon single sphere [-]

$\mathrm{c}_{\mathrm{d}, 0} \quad$ mean drag coefficient in the layer [-]

$\mathrm{c}_{\mathrm{dp}} \quad$ mean form resistance coefficient in the REV [-]

$\mathrm{c}_{\mathrm{FL}} \quad$ mean skin friction coefficient on the REV laminar region [-]

$\mathrm{c}_{\mathrm{p}} \quad$ specific heat $[\mathrm{J} /(\mathrm{kg} \mathrm{K})]$ 
$d_{c h} \quad$ character pore size in the cross section [m]

$\mathrm{d}_{\mathrm{p}} \quad$ particle diameter $[\mathrm{m}]$

f hydrodynamic resistance coefficient [-]

$\mathrm{f}_{1} \quad$ turbulent length scale [m]

g acceleration due to gravity $\left[\mathrm{m} / \mathrm{s}^{2}\right]$

$\mathrm{H} \quad$ height of the channel [m]

$\mathrm{h}=\mathrm{H} / 2$, half-width of the channel [m]

$\mathrm{h}_{\mathrm{r}} \quad$ pore scale microroughness layer thickness [m]

$\mathrm{S}_{\mathrm{w}}$ internal surface in the REV $\left[\mathrm{m}^{2}\right]$

满 turbulent eddy viscosity $\left[\mathrm{m}^{2} / \mathrm{s}\right]$

$\mathrm{K}_{\mathrm{ST}}$ thermal conductivity of solid phase [W/(m K)]

$\mathrm{K}_{\mathrm{T}} \quad$ turbulent eddy thermal conductivity [W/(m K)]

$\mathrm{K}_{\mathrm{w}} \quad$ similarity number in eddy viscosity boundary condition [-]

$\mathrm{k}_{\text {eff }} \quad$ effective thermal conductivity [(W/(m K)]

$\mathrm{k}_{\text {eff, } 0} \quad$ mean effective thermal conductivity in the layer $[(\mathrm{W} /(\mathrm{m} \mathrm{K})]$

$\mathrm{k}_{\mathrm{f}} \quad$ fluid thermal conductivity [W/(m K)]

$\mathrm{k}_{\mathrm{s}} \quad$ solid phase thermal conductivity [W/(m K)]

$\mathrm{k}_{0} \quad$ stagnant term, effective thermal conductivity [W/(m K)]

1 turbulence mixing length or molecular scale [m]

$\mathrm{m} \quad$ local porosity [-]

$\$ \mathrm{~m}$ i averaged porosity [-]

$\mathrm{m}_{0} \quad$ mean porosity in the layer [-]

$\mathrm{Nu}_{\mathrm{T}}=\frac{\alpha_{T} h}{\left(K_{T}+k_{f}\right)}$, turbulent Nusselt number [-]

$\mathrm{Nu}_{\mathrm{T}, 0} \quad$ mean turbulent Nusselt number in the layer [-]

$\mathrm{Pe} \quad=R e_{\psi} \operatorname{Pr}$, particle Peclet number [-]

$\mathrm{Pe}_{\mathrm{r}} \quad$ particle radius Peclet number (Eq. 39) [-]

$\mathrm{Pe}_{\mathrm{k}} \quad$ Brinkman screening length Peclet number (Eq. 40) [-]

$\operatorname{Pr} \quad=\varangle \mathrm{a}_{\mathrm{f}}$, Prandtl number [-]

$\operatorname{Pr}_{\mathrm{T}} \quad=\mathrm{K}_{\mathrm{m}} / \mathrm{K}_{\mathrm{T}}$, turbulent Prandtl number [-]

$\mathrm{p} \quad$ pressure $[\mathrm{Pa}]$ and pitch in regular porous $2 \mathrm{D}$ and $3 \mathrm{D}$ medium $[\mathrm{m}]$

$\mathrm{Q}_{0} \quad$ channel boundary heat flux $\left[\mathrm{W} / \mathrm{m}^{2}\right]$

$\mathrm{Re}_{\mathrm{ch}} \quad$ Reynolds number of pore hydraulic diameter [-]

$\operatorname{Re}_{\mathrm{R}}=\frac{d_{p} \tilde{u}}{\mathbf{v}}$, particle Reynolds number [-]

$\mathrm{S}_{\mathrm{w}} \quad$ specific surface of a porous medium $\quad \mathrm{S}_{\mathrm{w}} / \mathrm{S} \mathrm{S}[\mathrm{1} / \mathrm{m}]$ 
$\mathrm{S}_{\mathrm{wL}} \quad$ portion of specific surface bounding laminar flow [1/m]

$\mathrm{S}_{\mathrm{wm}} \quad$ mean specific surface $[1 / \mathrm{m}]$

$\left.\mathrm{S}_{\mathrm{wp}} \quad=\mathrm{S}_{\mathrm{z}} /\right) \mathrm{S}[1 / \mathrm{m}]$

$\mathrm{S}_{\mathrm{wT}} \quad$ portion of specific surface bounding turbulent flow $[1 / \mathrm{m}]$

$\mathrm{T}$ temperature $[\mathrm{K}]$

$\mathrm{T}_{\mathrm{a}} \quad$ reference temperature $[\mathrm{K}]$

$\mathrm{T}_{\mathrm{m}}=\frac{Q_{0}}{\mathrm{\rho}_{f} c_{p, f} u_{m}}$, characteristic convective fluid temperature across the porous layer $[\mathrm{K}]$

$\mathrm{T}_{\mathrm{w}} \quad$ wall temperature $[\mathrm{K}]$

$\mathrm{T}_{0} \quad$ mean temperature in the layer $[\mathrm{K}]$

$\mathrm{u} \quad$ velocity component in $\mathrm{x}$ direction $[\mathrm{m} / \mathrm{s}]$

$\mathrm{u}_{0} \quad$ mean velocity in the layer $[\mathrm{m} / \mathrm{s}]$

$\mathrm{u}_{\mathrm{m}} \quad=\left(-\frac{z_{m}}{\rho_{f}} \frac{\overrightarrow{d \vec{p}}}{d x}\right)^{1 / 2}$, characteristic velocity in the layer $[\mathrm{m} / \mathrm{s}]$

$\mathrm{u}_{\mathrm{r}_{\mathrm{rk}}} \quad$ friction velocity $[\mathrm{m} / \mathrm{s}]$

$\mathrm{x} \quad$ horizontal coordinate component [m]

y lateral coordinate component [m]

$\mathrm{z} \quad$ vertical coordinate component $[\mathrm{m}]$

\section{Subscripts}

f fluid phase

L laminar

r roughness

s solid phase

$\mathrm{T}$ turbulent

\section{Superscripts}

$\wedge \quad$ fluctuation of quantity about intrinsic average due to medium irregularity

\section{Operators}




$$
\begin{array}{ll}
\sim & =\frac{1}{\Delta \Omega_{f}} \int_{\Delta \Omega_{f}}() d \Omega \\
\circ \quad & \frac{1}{\Delta \Omega_{s}} \int_{\Delta \Omega_{s}}() d \Omega \\
<\mathrm{f}_{\mathrm{f}} \quad & \frac{1}{\Delta \Omega} \int_{\Delta \Omega_{f}}() d \Omega \\
<\mathrm{s}_{\mathrm{s}} & =\frac{1}{\Delta \Omega} \int_{\Delta \Omega_{s}}() d \Omega \\
<\quad & \quad<>_{\mathrm{f}}+<>_{\mathrm{s}}
\end{array}
$$

\section{Greek Letters}

'\# averaged heat transfer coefficient over $\mathrm{M}_{\mathrm{w}}\left[\mathrm{W} /\left(\mathrm{m}^{2} \mathrm{~K}\right)\right]$

'\#, mean heat transfer coefficient across the layer $\left[\mathrm{W} /\left(\mathrm{m}^{2} \mathrm{~K}\right)\right]$

$\mathrm{IZ}_{\mathrm{T}, \mathrm{m}}=\mathrm{\rho}_{f} c_{p, f} \mathcal{U}_{m}$, characteristic layer heat transfer coefficient $\left[\mathrm{W} /\left(\mathrm{m}^{2} \mathrm{~K}\right)\right]$

" ${ }_{\mathrm{w}}$ heat transfer coefficient at the wall $\left[\mathrm{W} /\left(\mathrm{m}^{2} \mathrm{~K}\right)\right]$

* micro-scale inhomogeneity [m]

8 friction coefficient in tubes [-]

$<\quad$ kinematic viscosity $\left[\mathrm{m}^{2} / \mathrm{s}\right]$

D density $\left[\mathrm{kg} / \mathrm{m}^{3}\right]$

$F_{b} \quad$ turbulent coefficient exchange ratio $\mathrm{K}_{\mathrm{m}} / \mathrm{K}_{\mathrm{b}}[-]$

$\mathrm{F}_{\mathrm{T}} \quad$ turbulent coefficient exchange ratio $\mathrm{K}_{\mathrm{m}} / \mathrm{K}_{\mathrm{T}}[-]$

) $\mathrm{S}$ representative elementary volume $(\mathrm{REV})\left[\mathrm{m}^{3}\right]$

) $\mathrm{S}_{\mathrm{f}}$ pore volume in a $\mathrm{REV}\left[\mathrm{m}_{3}\right]$

) $\mathrm{S}_{\mathrm{s}}$ skeleton volume in a $\operatorname{REV}\left[\mathrm{m}_{3}\right]$

\section{REFERENCES}

Aarov, M. A., Todes, O. M. and Narinskii, D. A., 1979, "Apparatuses with Stationary Granular 
Bed," Chemistry, Leningrad, p. 176.

Adnani, P., Raffray, A. R., Abdou, M. A. and Catton, I., 1989, "Modeling of Effective Thermal Conductivity for a Packed Bed," Department of Mechanical, Aerospace, and Nuclear Engineering, University of California, Los Angeles, UCLA-FNT-29, 50 pgs. (Mimeographed.)

Boothroyd, R. G., 1971, Flowing Gas-Solids Suspensions, Chapman and Hall Ltd., London, 1971.

Ergun, S., 1952, "Fluid Flow Through Packed Columns," Chemical Engineering Progress, Vol. 48, pp. 89-94.

Fand, R. M., Kim, B. Y., Lam, A. C. and Phan, R. T., 1987,"Resistance to the Flow of Fluids Through Simple and Complex Porous Media Whose Matrices Are Composed of Randomly Packed Spheres," ASME Journal of Fluids Engineering, Vol. 109, pp. 268-74.

Fand, R. M. and Thinakaran, R., 1990, "The Influence of the Wall on Flow Through Pipes Packed with Spheres," ASME Journal of Fluids Engineering, Vol. 112, No. 1, pp. 84-88.

Gratton, L. J., Travkin, V. S. and Catton, I., 1993, "Transport Coefficient Dependence upon Solid Phase Morphology for Single Phase Convective Transport in Porous Media," Heat Transfer in Porous Media, ASME HTD-Vol. 240, pp. 11-21.

Heat Exchanger Design Handbook, 1983, Hemisphere Publishing Corporation, Vol. 1,2.

Klenov, O. P. and Matros, Yu. Sh., 1990, "Effect of Loading Conditions on the Porosity and Hydraulic Resistance of a Stationary Granular Bed," Theoretical Foundations of Chemical Engineering, Vol. 24, No. 2, pp. 206-210.

Koch, D. L. and Brady J. F., 1985, "Dispersion in Fixed Beds," Journal of Fluid Mechanics, Vol. 154, pp. 399-427.

Kokorev, V. I., Subbotin, V. I., Fedoseev, V. N. et al., 1987, "On Interconnection of Hydraulic Resistance and Heat Transfer in Porous Media," High Temperature, Vol. 25, No. 1, pp. 82-87.

Kovalenko, Yu. A., 1991, "Thermal Conductivity in Disperse Porous Media: A Review of Modeling Results in Soviet Research," Russian Journal of Engineering Thermophysics, Vol. 1, pp. 225-43. 
Nikuradse, J., 1950, "Laws of Flow in Rough Pipes," NACA TM 1292.

Travkin, V. S., 1981, "Steady Free Convection in the Cavity With Variable Wall Temperature," Heat Exchange in Mono- and Bi-phase Media, Naukova Dumka, Kiev, pp.15-19.

Travkin, V.S., 1984, "Numerical Simulation of Heat and Mass Transfer in a Continuous-Casting Mold," High Temperature, Vol.22, No.2, pp.251-258.

Travkin, V.S., 1985, "Numerical Integration of Free Convection Compressible Gas Equation in Enclosed Cylindrical Cavity on Conjugated Statement," Numerical Methods of Continuous Medium Mechanics, Vol.16, No.3, pp.128-152.

Travkin, V.S., 1987, "Mathematical Simulation of Turbulent Diffusion of Admixtures in Boundary Layer Over Rough Surfaces," Ph.D. Dissertation Abstracts, Kiev State University, Kiev, U.S.S.R.

Travkin, V. and Catton, I., 1992a,"Turbulent Forced Transport Governing Equations in High Permeability Medium," submitted to the Physics of Fluids, Sec A.

Travkin, V. and Catton, I., 1992b, "Models of Turbulent Thermal Diffusivity and Transfer Coefficients for a Regular Packed Bed of Spheres," Proceedings, 28th National Heat Transfer Conference, San Diego, CA, ASME, HTD-Vol. 193, pp.15-23.

Travkin, V. and Catton, I., 1992c, "The Numerics of Turbulent Processes in High Porosity Nonuniform Porous Media," Proceedings, SIAM 40th Anniversary Meeting, Los Angeles, CA, pp.A36.

Travkin, V. S. and Catton, I., 1995, "A Two-Temperature Model for Turbulent Flow and Heat Transfer in a Porous Layer," Journal of Fluids Engineering, Vol. 117, pp. 181-188.

Tsuji, Y., Morikawa, Y. and Fujiwara, Y., 1985, "Pipe Flow with Solid Particles Fixed in Space," International Journal of Multi-phase Flow, Vol. 11, No. 2, pp. 177 - 188.

Watanabe, H., 1989, "Drag Coefficient and Voidage Function on Fluid Flow through Granular Packed Beds," International Journal of Engineering Fluid Mechanics, Vol. 2, No. 1, pp. 93-108. 